\title{
Dynamics of Argumentation Systems: A Basic Theory
}

\author{
Beishui Liao ${ }^{1}, \mathrm{Li}_{\mathrm{Jin}}{ }^{1}$ and Robert C. Koons ${ }^{2}$ \\ 1 Zhejiang University \\ Hangzhou, Zhejiang, China \\ \{baiseliao,jinli-pearl\}@zju.edu.cn \\ 2 University of Texas at Austin \\ Austin, Texas, U.S.A. \\ koons@phil .utexas.edu
}

\begin{abstract}
The changing of arguments and their attack relation is an intrinsic property of a variety of argumentation systems. So, it is very important to efficiently figure out how the status of arguments in a system evolves when the system is updated. However, unlike other areas of argumentation that have been deeply explored, such as argumentation semantics, proof theories, and algorithms, etc., dynamics of argumentation systems has been comparatively neglected. In this paper, we introduce a general theory (called a division-based method) to cope with this problem based on a new concept: the division of an argumentation framework. When an argumentation framework is updated, it is divided into three parts: an unaffected, an affected, and a conditioning part. The status of arguments in the unaffected sub-framework remains unchanged, while the status of the affected arguments is computed in a special argumentation framework (called a conditioned argumentation framework, or briefly CAF) that is composed of an affected part and a conditioning part. We have proved that under a certain semantics that satisfies the directionality criterion (complete, preferred, ideal, or grounded semantics), the extensions of the updated framework are equal to the result of a combination of the extensions of an unaffected sub-framework and sets of the extensions of a set of assigned CAFs. Due to the efficiency of the division-based method, it is expected to be very useful in various kinds of argumentation systems where arguments and attacks are dynamics.
\end{abstract}

\section{Introduction}

Argumentation is a non-monotonic logic for reasoning with incomplete and uncertain knowledge [10, 4]. The changing of arguments and attack relations is a very common property of various kinds of argumentation systems, especially argumentation-based deliberative agents within a dynamic environment, including belief revision, deliberation, and decision making, etc 11, 7, 12, 15, 18. These researches show that in many argumentation systems, arguments and their attack relations evolve with the changing of the underlying knowledge. However, as Dunne and Wooldridge pointed out in [11, "..., the treatment of algorithms and complexity issues relating to determining argument status in dynamically changing environments has been somewhat neglected".

With the changing of arguments and/or attack relations of an argumentation system, the status of some arguments changes, while that of others remains untouched. Now, one of the challenging problems is how to efficiently compute the dynamics of argumentation systems. When an argumentation system is modified, we may simply recompute the status of each argument afresh. However, this method is obviously inefficient, and in most of cases, difficult. This is due to the fact that under most argumentation semantics, such as complete semantics, 
preferred semantics, and ideal semantics, etc., an argumentation framework may have several extensions, i.e., each argument may have several possible statuses. As a result, the computation of the status of arguments under these argumentation semantics [1] is often intractable.

To cope with this problem, there have been a small number of efforts 1, 5, 9. First, Boella et al studied the dynamics of argumentation by exploring the principles according to which the extension does not change when the set of arguments or the attack relations between them are changed [5, 6. However, they have not considered how the extensions of an argumentation system evolve when new arguments are added or the old ones are removed. Second, Cayrol et al addressed the problem of revising the set of extensions of an abstract argumentation system, and studied how the extensions of an argumentation system may evolve when a new argument is received [9]. However, they restricted their study to the case of adding just one argument having only one interaction with an initial argument. Third, Amgoud et al used dynamics of argumentation in the decision-making of an autonomous agent [1. They studied how the acceptability of arguments evolves when the decision system is extended by new arguments without computing the whole extensions. However, they also considered the situation where only one argument is added to the system. In addition, all of these existing researches have not studied the time complexity of computing the dynamics of argumentation.

According to the above analysis, a more general theory is needed to formulate the dynamics of argumentation systems, with the following three characteristics: (i) the number of arguments and attack relations to be added to (or deleted from) an argumentation system is unlimited;

(ii) both the addition and the deletion of arguments and/or attack relations are considered, and

(iii) the time complexity of computing the dynamics of argumentation systems is explored.

\section{The basic idea of the division-based method}

This work is based on Dung's abstract argumentation framework [10], which is defined as a tuple $A F=\langle A, R\rangle$, where $A$ is a finite set of arguments, and $R \subseteq A \times A$ is a set of attack relations. Given an argumentation framework, a fundamental problem is to determine which arguments can be considered justified. According to 10, extension-based argumentation semantics is a formal way to answer this question. Here, an extension represents a set of arguments that are considered to be acceptable together, which is based on the following three important notions: conflict-free, acceptability, and admissible set. A set $B \subseteq A$ of arguments is conflict-free if and only if $\nexists \alpha, \beta \in B$, such that $(\alpha, \beta) \in R$. An argument $\alpha \in A$ is acceptable w.r.t. a set $B \subseteq A$ of arguments, if and only if $\forall \beta \in A$, if $(\beta, \alpha) \in R$, then $\exists \gamma \in B$, such that $(\gamma, \beta) \in R$. A conflict-free set of arguments $B \subseteq A$ is admissible if and only if each argument in $B$ is acceptable w.r.t. $B$. According to these notions, it is said that: (1) an admissible set of arguments $B \subseteq A$ is a complete extension if and only if each argument that is acceptable w.r.t. $B$ is in $B$; (2) $B$ is a preferred extension if and only if $B$ is a maximal complete extension; (3) $B$ is a grounded extension if and only if $B$ is the minimal complete extension; and (4) $B$ is ideal if and only if $B$ is admissible and it is contained in every preferred set of arguments. The ideal extension is the maximal (w.r.t. set-inclusion) ideal set. We use $\mathcal{E}_{\mathcal{C O}}(A F), \mathcal{E}_{\mathcal{P R}}(A F), \mathcal{E}_{\mathcal{G R}}(A F)$ and $\mathcal{E}_{\mathcal{I D}}(A F)$ to denote the set of complete, preferred, grounded, and ideal extensions of $A F$, respectively. Meanwhile, for convenience, we use $\mathcal{S} \in\{\mathcal{C O}, \mathcal{P} \mathcal{R}, \mathcal{G R}, \mathcal{I D}\}$ to denote one of the four argumentation semantics. Given $A F=\langle A, R\rangle$ and $E \in \mathcal{E}_{\mathcal{S}}(A F)$, the set of arguments $A$ can be partitioned into three parts: $\mathscr{A}(A F, E), \mathscr{R}(A F, E)$ and $\mathscr{U}(A F, E)$, where $\mathscr{A}(A F, E)=E$ is a set of accepted arguments w.r.t. $E ; \mathscr{R}(A F, E)=\{\alpha \in A \mid \exists \beta \in E$, such that $(\beta, \alpha) \in R\}$ is a set of rejected arguments w.r.t. $E$; and $\mathscr{U}(A F, E)=A \backslash(\mathscr{A}(A F, E) \cup \mathscr{R}(A F, E))$ is a set of undecided arguments w.r.t. $E$. A 3-tuple $\mathrm{S}(A F, E)=(\mathscr{A}(A F, E), \mathscr{R}(A F, E), \mathscr{U}(A F, E))$ is 
called a status assignment of an argumentation framework $A F$ w.r.t. $E$.

Based on the above argumentation theory, the next step is to compute the extensions of a given argumentation framework (static or dynamic). However, this task is still challenging: there exist no polynomial time algorithms to compute the extensions of a given argumentation framework under most semantics. In order to improve the computational efficiency, when an argumentation framework is updated, a natural idea is to use the computational results that have been obtained. According to the notion directionality of argumentation [3], the status of an argument under the semantics that satisfy the directionality criteria is affected only by the status of its defeaters. So, when a set of arguments and/or a set of attack relations are added to (or deleted from) an argumentation framework, we will be able to identify a set of arguments that are affected and a set of arguments that are unaffected. As a result, the status of arguments in the sub-framework corresponding to the unaffected part is obtained directly. Now, the challenging problems include the following two aspects: (1) how to compute the extensions of the sub-framework corresponding to the affected part, and (2) how to combine the extensions of the two sub-frameworks to get the extensions of the updated framework.

The first problem is resolved by introducing a special kind of argumentation framework, called conditioned argumentation framework (corresponding to the affected sub-framework in this paper), in which the status of arguments is dependent on the status of arguments outside the argumentation framework.

Given an argumentation framework $A F_{1}=\left\langle A_{1}, R_{1}\right\rangle$, a conditioned argumentation framework with respect to $A F_{1}$ is a tuple $C A F=\left(\left\langle A_{2}, R_{2}\right\rangle,\left(C\left(A_{1}\right), \mathcal{I}_{\left(C\left(A_{1}\right), A_{2}\right)}\right)\right)$, in which: $\left\langle A_{2}, R_{2}\right\rangle$ is an argumentation framework that is conditioned by $A F_{1}$, in which $A_{2} \cap A_{1}=\emptyset ; C\left(A_{1}\right) \subseteq A_{1}$ is a nonempty set of arguments (called conditioning arguments) that have interactions with arguments in $A_{2}$, i.e., $\forall \alpha \in C\left(A_{1}\right), \exists \beta \in A_{2}$, such that $(\alpha, \beta) \in \mathcal{I}_{\left(C\left(A_{1}\right), A_{2}\right)}$; and $\mathcal{I}_{\left(C\left(A_{1}\right), A_{2}\right)} \subseteq$ $C\left(A_{1}\right) \times A_{2}$ is a set of attack relations.

The status of arguments in $C\left(A_{1}\right)$ of a CAF is assigned within $A F_{1}$, an argumentation framework independent of $\left\langle A_{2}, R_{2}\right\rangle$. So, after the status of arguments in $C\left(A_{1}\right)$ is assigned according to a specific extension $E \in \mathcal{E}_{\mathcal{S}}\left(A F_{1}\right)$, we call the $\mathrm{CAF}$ an assigned $\mathrm{CAF}$, denoted as $C A F[E]=\left(\left\langle A_{2}, R_{2}\right\rangle,\left(C\left(A_{1}\right)[E], \mathcal{I}_{\left(C\left(A_{1}\right), A_{2}\right)}\right)\right)$, in which $C\left(A_{1}\right)[E]=\operatorname{def}_{\text {def }}\left(\mathscr{A}\left(A F_{1}, E\right) \cap\right.$ $\left.C\left(A_{1}\right), \mathscr{R}\left(A F_{1}, E\right) \cap C\left(A_{1}\right), \mathscr{U}\left(A F_{1}, E\right) \cap C\left(A_{1}\right)\right)$ is a set of arguments with assigned statuses, called a condition.

The acceptability semantics of the assigned CAF is defined as follows: A set $B \subseteq A_{2}$ of arguments is conflict-free if and only if $\nexists \alpha, \beta \in B$, such that $(\alpha, \beta) \in R_{2}$. An argument $\alpha \in A_{2}$ is acceptable w.r.t. a set $B \subseteq A_{2}$ of arguments under the condition $C\left(A_{1}\right)[E]$, if and only if the following two conditions hold: $(1) \forall \beta \in A_{2}$, if $(\beta, \alpha) \in R_{2}$, then $\exists \gamma \in B$, such that $(\gamma, \beta) \in R_{2}$, or $\exists \xi \in C\left(A_{1}\right)$, such that $\xi$ is accepted w.r.t. $E$ and $(\xi, \beta) \in \mathcal{I}_{\left(C\left(A_{1}\right), A_{2}\right)}$; and $(2) \forall \beta \in C\left(A_{1}\right)$, if $(\beta, \alpha) \in \mathcal{I}_{\left(C\left(A_{1}\right), A_{2}\right)}$, then $\beta$ is rejected w.r.t. $E_{1}$. A conflict-free set $B \subseteq A_{2}$ of arguments is admissible if and only if each argument in $B$ is acceptable w.r.t. $B$ under the condition $C\left(A_{1}\right)[E]$. An admissible set of arguments $B \subseteq A_{2}$ is a complete extension if and only if each argument that is acceptable w.r.t. $B$ under the condition $C\left(A_{1}\right)[E]$ is in $B$. $B$ is a preferred extension if and only if $B$ is a maximal complete extension. $B$ is a grounded extension if and only if $B$ is the minimal complete extension. $B$ is ideal if and only if $B$ is admissible and it is contained in every preferred set of arguments. The ideal extension is the maximal (w.r.t. set-inclusion) ideal set.

After computing the extensions of affected sub-framework according to the semantics of assigned CAF, they are combined with the extensions of the unaffected sub-framework to form the extensions of the original updated framework. The definition of combining the extensions of two sub-frameworks (affected and unaffected) was formally presented in [13]. 
Finally, we have proved that under the argumentation semantics that satisfy the directionality criteria (including complete, grounded, preferred and ideal semantics), the extensions of an updated framework are the same as the result of a combination of the extensions of an unaffected sub-framework and sets of the extensions of a set of assigned CAFs 13 .

\section{Conclusions}

The contributions of the division-based method introduced in this paper mainly include the following aspects:

1. Propose a theory to divide an argumentation framework: by taking advantage of the characteristics of the relationship between the status of arguments and their attack relation, we have formally described a theory of dividing an argumentation framework and proved the correctness of this division.

2. Propose a theory to compute the status of affected arguments: we have introduce two new concepts, conditioned argumentation framework (CAF) and assigned CAFs, as well as the semantics of an assigned CAF, based on which the status of affected arguments can be computed correctly.

3. Propose a theory to combine the semantics of the two kinds of sub-frameworks: we have formally defined the semantics combination of the two kinds of sub-frameworks, and proved the soundness and completeness of the combination. Meanwhile, we have proved the correctness of status evolution of arguments by using the division-based method.

With the above contributions, the division-based method proposed in this paper has the following characteristics:

1. Generality: It is a general theory in the sense of the following two aspects. First, this theory is applicable to various kinds of argumentation semantics that satisfy the directionality criterion, including complete semantics, preferred semantics, grounded semantics, and ideal semantics, etc. Second, this theory is able to treat with a general form of dynamics of argumentation, i.e., (i) the number of arguments and attacks to be added to or deleted from an argumentation system is unlimited; and (ii) both the addition and the deletion of arguments and/or attacks are applicable.

2. Efficiency: Qualitatively, it is obvious that in most cases (although not in all cases) the division-based method is more efficient. This is mainly due to the following two reasons. First, there exist linear time algorithms for the division of an argumentation framework (in that the problem of dividing an argumentation framework corresponds to finding the nodes reachable from a set of nodes in a directed graph). Second, when computing the extensions of a modified argumentation framework, we may reuse some previous computation, rather than simply recompute the status of each argument afresh.

With the above two characteristics, this theory is expected to be very useful in various kinds of argumentation-based systems, especially belief revision, deliberation, decision-making, and negotiation, within agents and multi-agent systems. The reason is that in these systems, underlying knowledge and information are often uncertain, incomplete, inconsistent, and everchanging. As a result, the corresponding argumentation systems are dynamic by nature. So, the efficient division-based method will facilitate the development of these systems. 


\section{Acknowledgments}

We are grateful to the anonymous referees for their valuable comments. This work was partially supported by the National Science Foundation of China (No.60773177) and the Zhejiang Provincial Natural Science Foundation of China (No.Y1100036).

\section{References}

[1] L.Amgoud, S.Vesic, On Revising Argumentation-Based Decision Systems, In: Proc. of ECSQARU 2009, LNAI 5590, 2009, pp. 71-82.

[2] L.Amgoud.A Unified Setting for Inference and Decision: An Argumentation-based Approach. In: Proc. of UAI 2005, pp. 26-33.

[3] P. Baroni, M. Giacomin, On principle-based evaluation of extension-based argumentation semantics, Artificial Intelligence 171 (2007) 675-700.

[4] T.J.M. Bench-Capon, P. E. Dunne, Argumentation in artificial intelligence. Artificial Intelligence 171 (2007) 619-641.

[5] G.Boella, S.Kaci, L.van der Torre, Dynamics in Argumentation with Single Extensions: Abstraction Principles and the Grounded Extension, In: Proc. of ECSQARU 2009, LNAI 5590, 2009, pp. 107-118.

[6] Guido Boella, Souhila Kaci, Leendert van der Torre: Dynamics in argumentation with single extensions: attack refinement and the grounded extension, In: Proc. of AAMAS 2009, 2009, 1213-1214.

[7] M.Capobianco, C.I.Chesnevar, G.R.Simari, Argumentation and the Dynamics of Warranted Beliefs in Changing Environments, JAAMAS 11(2) (2005) 127-151.

[8] D.V.Carbogim, Dynamics on Formal Argumentation, Ph.D Thesis, University of Edinburgh, 2000.

[9] C.Cayrol, F.D.de St-Cyr, F.Dupin, M.Lagasquie-Schiex, Change in Abstract Argumentation Frameworks: Adding an Argument, Journal of Artificial Intelligence Research 84(2010) 49-84.

[10] P.M.Dung, On the acceptability of arguments and its fundamental role in nonmonotonic reasoning, logic programming and n-person games, Artificial Intelligence 77 (1995) 321-357.

[11] P.E.Dunne, M.Wooldridge, Complexity of Abstract Argumentation, in: I. Rahwan, G. R. Simari (eds.), Argumentation in Artificial Intelligence, Springer, 2009, pp.85-104.

[12] B.Liao, H.Huang, ANGLE: An Autonomous, Normative and Guidable Agent with Changing Knowledge, Information Sciences 180 (2010) 3117-3139.

[13] B.Liao, L.Jin, R.C.Koons, Dynamics of Argumentation Systems: A Division-Based Method, submitted to Artificial Intelligence Journal.

[14] S.Modgil, M.Caminada, Proof Theories and Algorithms for Abstract Argumentation Frameworks. In: I. Rahwan, G. R. Simari (eds.), Argumentation in Artificial Intelligence, Springer, 2009: 105129.

[15] M.O.Moguillansky, et al, Argument Theory Change Applied to Defeasible Logic Programming, In:Proc.of AAAI-08,2008, pp.132-137.

[16] K.Okuno, K.Takahashi, Argumentation System with Changes of an Agent's Knowledge Base, In: Proc. of IJCAI-09, 2009, pp. 226-232.

[17] H.Prakken, Relating protocols for dynamic dispute with logics for defeasible argumentation, Synthese 127 (2001) 187-219.

[18] N.D.Rotstein, et al, An Abstract Argumentation Framework for Handling Dynamics, In: Proc. of NMR2008, 2008, pp. 131-139. 